\title{
Towards real-world capable spatial memory in the LIDA cognitive architecture
}

\author{
Tamas Madl ${ }^{\text {a,b, }}{ }^{*}$, Stan Franklin ${ }^{c}$, Ke Chen ${ }^{a}$, Daniela Montaldi ${ }^{d}$, \\ Robert Trappl ${ }^{\mathrm{b}}$
}

a School of Computer Science, University of Manchester, Manchester M13 9PL, UK
b Austrian Research Institute for Artificial Intelligence, Vienna A-1010, Austria
${ }^{\mathrm{c} I n s t i t u t e ~ f o r ~ I n t e l l i g e n t ~ S y s t e m s, ~ U n i v e r s i t y ~ o f ~ M e m p h i s, ~ M e m p h i s, ~ T N ~ 38152, ~ U S A ~}$
${ }^{\mathrm{d}}$ School of Psychological Sciences, University of Manchester, Manchester M13 9PL, UK

Received 30 September 2015; received in revised form 11 February 2016; accepted 15 February 2016

\section{KEYWORDS}

Spatial memory;

LIDA;

Cognitive architecture;

Computational cognitive modeling

\begin{abstract}
The ability to represent and utilize spatial information relevant to their goals is vital for intelligent agents. Doing so in the real world presents significant challenges, which have so far mostly been addressed by robotics approaches neglecting cognitive plausibility; whereas existing cognitive models mostly implement spatial abilities in simplistic environments, neglecting uncertainty and complexity.

Here, we take a step towards computational software agents capable of forming spatial memories in realistic environments, based on the biologically inspired LIDA cognitive architecture. We identify and address challenges faced by agents operating with noisy sensors and actuators in a complex physical world, including near-optimal integration of spatial cues from different modalities for localization and mapping, correcting cognitive maps when revisiting locations, the structuring of complex maps for computational efficiency, and multi-goal route planning on hierarchical cognitive maps. We also describe computational mechanisms addressing these challenges based on LIDA, and demonstrate their functionality by replicating several psychological experiments.
\end{abstract}

(c) 2016 Elsevier B.V. All rights reserved.

\footnotetext{
* Corresponding author at: Austrian Research Institute for Artificial Intelligence, Vienna A-1010, Austria.

E-mail address: tamas.madl@gmail.com (T. Madl).
} 


\section{Introduction}

Spatial representations are important for biological and artificial agents, to help them localize and navigate to important objects and places (such as food sources or shelters). Current computer models for learning spatial representations either neglect cognitive plausibility in favour of performance, such as simultaneous localization and mapping (SLAM) in robotics, or are incapable of running in large-scale, complex, uncertain environments perceived through noisy sensors.

Since biological cognition has been shaped by the structure, constraints, and challenges of the physical world, we argue that cognitive architectures should take these into account, as well. This argument is in accordance with the roadmap for the BICA Challenge, which also places importance on real-life capability (Samsonovich, 2012).

This paper describes an effort to take the LIDA (Learning Intelligent Distribution Agent) cognitive architecture (Franklin, Madl, D’Mello, \& Snaider, 2014) closer to this goal. We hypothesize and implement approaches to tackle the sensory noise, uncertainty, and complexity of realistic environments. We also introduce a novel, conceptual and partially implemented, hierarchical spatial memory model, inspired by the neural basis of spatial cognition in brains, and provide a preliminary interface to realistic environments via the Robot Operating System (ROS) (Quigley et al., 2009). We demonstrate these extensions to LIDA in three-dimensional simulated environments that include simulated physics and high-quality graphics, based on the Player/Stage/Gazebo simulator. ${ }^{1}$ This simulator presents the same interface to the agent as real devices, and an agent able to control a robot in Gazebo is also able to control the same robot in similar environments in the real world, without any changes to the control code (Rusu, Maldonado, Beetz, \& Gerkey, 2007).

We build on and integrate our previous work investigating biologically and cognitively plausible implementations of Bayesian localization (Madl, Franklin, Chen, Montaldi, \& Trappl, 2014), Bayesian nonparametric clustering for map structuring (Madl, Franklin, Chen, Trappl, \& Montaldi, submitted for publication), and route planning based on activation gradients $^{2}$ (Madl, Franklin, Chen, \& Trappl, 2013). The method for cognitive map correction (loop closing) is presented for the first time below. Although based on established mathematical tools from robotics, it is - to our knowledge - the first mechanism for large-scale cognitive map correction implementable in brains, and consistent with the replay phenomena observed in the rodent hippocampus (Carr, Jadhav, \& Frank, 2011).

The present work is also (to our knowledge) the first to provide implementations of these mechanisms in a both cognitively and biologically plausible fashion (fitting behaviour data and implementable in brains), and integrated

\footnotetext{
1 http://www.gazebosim.org/.

2 Route planning in navigation space based on activation gradients has been proposed before (Burgess, Jackson, Hartley, \& O'keefe, 2000; Schölkopf \& Mallot, 1995), but not on a hierarchy - as it is in this work - which significantly improves its performance on multigoal problems.
}

within the same cognitive architecture. Further contributions include concrete implementations of some features listed in the BICA Table (Samsonovich, 2010) which until now were only part of conceptual LIDA, including basic stereo colour vision, a cognitive map, spatial learning, and fusing information from multiple types of sensors and modalities via Bayesian update.

\section{Related work}

Apart from the complex perception problem, the most challenging problems for building spatial representations in realistic environments include localization and mapping under sensory noise, and correcting incorrect representations when revisiting known locations (loop closing). The robotics community has developed several solutions to these problems - see Bailey and Durrant-Whyte (2006), DurrantWhyte and Bailey (2006), Thrun and Leonard (2008), and Williams et al. (2009). They have been designed to be accurate, not cognitively or biologically plausible, and rely on mechanisms that are difficult to implement in brains (e.g., many iterations performing operations on large matrices).

An exception is the partially connectionist RatSLAM system (Milford, Wyeth, \& Rasser, 2004), which can learn robust maps in outdoor environments (Prasser, Milford, \& Wyeth, 2006), and close large loops successfully if extended by a sophisticated data association method (Glover, Maddern, Milford, \& Wyeth, 2010). Parts of it have been argued to be biologically plausible (Milford, Wiles, \& Wyeth, 2010). However, RatSLAM has two disadvantages in the context of a cognitive model with long-term learning aiming for plausibility: (1) route planning only works along established routes (novel detours or shortcuts have not been demonstrated), (2) learned spatial information is mapped to a finite structure (attractor network) of fixed size which cannot be expanded.

On the other hand, models that emphasize plausibility cognitive architectures and plausible spatial memory models - mostly focus on simplistic simulated environments, usually with no sensory noise and limited size/complexity. There are a few neurally inspired spatial memory models that can deal with a limited amount of uncertainty and noise (Barrera, Cáceres, Weitzenfeld, \& Ramirez-Amaya, 2011; Burgess et al., 2000; Strösslin, Sheynikhovich, Chavarriaga, \& Gerstner, 2005), but have only been tested in small indoor environments. See Madl, Chen, Montaldi, and Trappl (2015) for a review.

\section{Spatial memory in brains}

Spatial memory encodes, stores and recalls spatial information about the environment and the self-location of agents (biological or artificial), which they need to keep track of to navigate successfully. In most mammals, keeping track of position is achieved by path integration, which refers to updating the agent's position based on a fixed point and the estimated movement trajectory (based on information from proprioceptive and vestibular systems as well as sensory flow (Fortin, 2008; Mittelstaedt \& Mittelstaedt, 1980)). It is a noisy process that accumulates large errors if uncorrected (Etienne, Maurer, \& Sguinot, 1996). 
Spatial information can be encoded in an egocentric fashion - relative to the agent's body and head direction - or as allocentric representations, relative to environmental landmarks/boundaries. Here, we will describe major brain areas associated with these representations and their correspondences in LIDA. For reasons of space, these descriptions will be very brief. More detail can be found in Madl et al. (2015).

The ability to recognize objects (e.g. landmarks, shelters, food sources, etc.) is a prerequisite for encoding useful spatial memories. The brain areas involved in this complex functionality include the sensory cortices and the areas marked (1a) and (1b) in Fig. 1 (Davachi, Mitchell, \& Wagner, 2003; Kiani, Esteky, Mirpour, \& Tanaka, 2007; Wilson et al., 2013; Winters \& Bussey, 2005). The recognition of places is associated with its own area in the parahippocampal cortex, often called the parahippocampal place area (PPA) (Epstein, 2008).

Neural representations of allocentric (world-centered) information in mammalian brains include place cells in the hippocampus, which represent spatial locations, firing only in small spatially constrained areas in an environment (ranging from $20 \mathrm{~cm}$ or less to several meters in diameter, Kjelstrup et al., 2008; O'Keefe \& Burgess, 1996). They also participate in associating objects with specific places (Kim, Delcasso, \& Lee, 2011; Manns \& Eichenbaum, 2009).

In these cells, 'hippocampal replay' has been observed a sequence of place cells associated with visited locations is frequently reactivated (replayed) either in the same order or in reverse, on rapid (sub-second) timescales, suggested to aid memory consolidation (Carr et al., 2011). When an animal runs along a track with a food source at the end, replay often occurs in reverse at the end of a run, and forward when anticipating a run; and it contains distance information between intersecting firing fields (Diba \& Buzsáki, 2007). Head direction is encoded by cells in a network including the anterior thalamic nuclei, mamillary body, subiculum and EC (Taube, 2007). Border cells (Lever, Burton, Jeewajee, O Keefe, \& Burgess, 2009; Solstad, Boccara, Kropff, Moser, \& Moser, 2008) and boundary vector cells (BVCs) (Barry et al., 2006; Burgess, 2008) in the subiculum play a role in representing the distance (and, for BVCs, the direction) to boundaries in the environment. Path integration, i.e. maintaining a location estimate by integrating self-motion signals, is performed by grid cells in the medial EC (Hafting, Fyhn, Molden, Moser, \& Moser, 2005; McNaughton, Battaglia, Jensen, Moser, \& Moser, 2006).

Together, these cell types form a core part of the 'cognitive map', i.e. a map-like allocentric representation of the environment (Burgess, 2008; McNaughton et al., 2006); and allow animals to keep track of where they are (place cells and grid cells), which direction they are facing (head direction cells), and where boundaries (border cells/BVCs) and objects (place cells) might be in their vicinity (see markers $2 a-2 c$ in Fig. 1).

In addition to allocentric representations, there are multiple egocentric brain areas encoding spatial information relative to the animal. These include the visual and auditory systems and the precuneus ( $(3)$ in Fig. 1), which is the main brain area concerned with egocentric representations and their use in controlling body and limb-centered actions (Kravitz, Saleem, Baker, \& Mishkin, 2011; Vogeley et al.,
2004; Zaehle et al., 2007) (for example, area 5d within the precuneus encodes 'reach vectors' between hand and target). The retrosplenial cortex (RSC) is involved with converting between egocentric and allocentric representations (Epstein, 2008) (marker 4 in Fig. 1). Finally, the basal ganglia (marker 5 in Fig. 1) encode guidance behaviours by means of associating spatial relations relative to the animal with actions (e.g., turn right at the rock). This is an effective strategy for well-known routes (Hartley, Maguire, Spiers, \& Burgess, 2003); however, allocentric representations ('cognitive maps') are required in order to be able to plan novel routes or shortcuts.

\section{Hypotheses}

The LIDA cognitive architecture is based on Global Workspace Theory (GWT) (Baars, 2002; Baars \& Franklin, 2009), an empirically supported theory of consciousness (Baars, Franklin, \& Ramsoy, 2013), and has been argued to be biologically plausible (Franklin, Strain, Snaider, McCall, \& Faghihi, 2012, 2014). Just as the rest of LIDA can be mapped on to the underlying neuroscience (Franklin et al., 2012) (although not always in a one-to-one fashion), it is also the aim of the model proposed here to have parts that functionally correspond to the relevant areas of the brain representing space. This imposes some functional and connectivity constraints.

Apart from well-established implications of the neural representations in these brain areas, including the existence of a neural path integrator (McNaughton et al., 2006) and cells representing current location (hippocampal 'place cells' (Moser, Kropff, \& Moser, 2008)), the spatial memory model presented here also proposes and requires the following hypotheses. They are motivated by computational challenges facing agents operating in the real world - the ability to represent uncertainty, to estimate locations based on uncertain data, and to represent large amounts of spatial information efficiently are all essential for a real-life, embodied cognitive agent. Our choice of computational approaches (among all possible mechanisms) directly follow from these hypotheses.

1. Spatial uncertainty is encoded in brains, and spatial cues are integrated in an approximately Bayes-optimal fashion. The representation of uncertainty is a computational requirement for localization in the real world, given the unavoidable sensory inaccuracies and noise, and it implies the existence of a mechanism for combining modalities with different accuracies. Apart from behavioural evidence substantiating such a mechanism (Cheng, Shettleworth, Huttenlocher, \& Rieser, 2007), we have found neural evidence based on single-cell recordings of rat hippocampal place cells in previous work, implying that these cells are able not only to represent but also to combine information from different modalities and the associated uncertainties (Madl et al., 2014).

2. Hippocampal replay (Carr et al., 2011) in awake mammals aids correcting cognitive maps based on revisited places (see Section 'Loop closing - fixing previously learned maps'). Despite local error correction by 

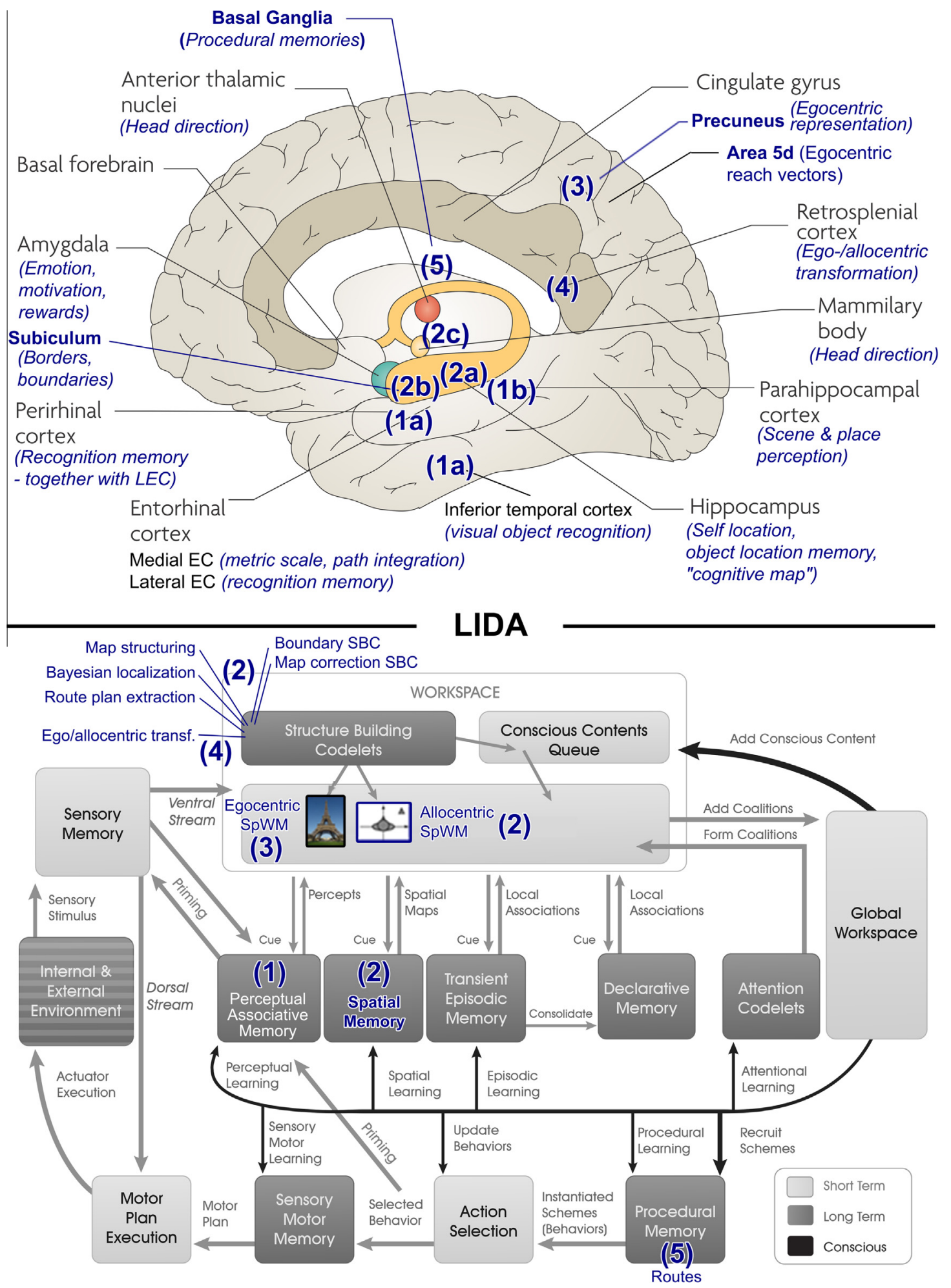

Fig. 1 Spatially relevant brain areas and LIDA modules. Top: Neural correlates involved in spatial processing. Modified from Bird and Burgess (2008) with permission. Bottom: functionally corresponding modules and processes in LIDA. Only spatially relevant correspondences are marked here; see Franklin et al. (2012, 2014) for others.

integrating spatial information, residual errors still accumulate. This can lead to incorrect maps and to duplicate representations of the same places. Thus, a mechanism is required that can close loops and correct maps when revisiting places.
3. Instead of a single unitary and global map, cognitive maps are fragmented (Derdikman \& Moser, 2010) and hierarchical (Hirtle \& Jonides, 1985), and their structure arises from clustering, i.e. from a process grouping together objects that are 'close' in some psychological 
space. Hierarchical representations are ubiquitous in computer science and robotics, given their efficiency in terms of access and search time and memory use. These advantages are important for storing and accessing largescale cognitive maps. We found evidence for hierarchies and a clustering mechanism accounting for them in Madl et al. (submitted for publication).

4. Human multi-goal route planning is consistent with a simple navigation strategy based on spreading activation on a recurrently interconnected, hierarchical, grid-like network of nodes representing locations (see Section 'Spatial extensions to LIDA - overview', Supplementary Information, and Madl et al., 2013).

\section{The LIDA cognitive architecture}

Here, we will briefly introduce LIDA - see Franklin et al. (2012, 2014) for a more detailed description of LIDA and its relationship to the brain. The LIDA cognitive architecture is based on prevalent cognitive science and neuroscience theories (e.g., Global Workspace Theory, situated cognition, and perceptual symbol systems - see Baars \& Franklin (2009)), and is one of the few cognitive models that are biologically plausible and to provide a plausible account for consciousness (Baars \& Franklin, 2009; Baars et al., 2013), attention, feelings and emotions; and has been partially implemented (Franklin et al., 2014; Goertzel, Lian, Arel, de Garis, \& Chen, 2010; Snaider, McCall, \& Franklin, 2011).

Similarly to the action-perception cycle in neuroscience (Freeman, 2002; Fuster, 2002), LIDA's cognitive cycle has the purpose of selecting an action based on percepts (Fig. 1 bottom). During each cycle, the LIDA agent senses its environment, stores information in Sensory Memory, and tries to recognize familiar objects, which are represented as nodes in Perceptual Associative Memory (PAM). It associates percepts with memories (declarative, episodic, spatial) recalled from a Sparse Distributed Memory (SDM) instance, creating models of the current situation (CSM) in the Workspace, which consist of the relevant PAM nodes copied to the Workspace. Several Structure Building Codelets ${ }^{3}$ (SBC) - specialized 'processors' - operate on the pre-conscious representations in the Workspace. Subsequently, Attention Codelets form coalitions with salient pre-conscious representations, which are moved to Global Workspace and compete for consciousness. The most salient ${ }^{4}$ representations are broadcast consciously, which enables the agent to choose actions applicable in the current situation from Procedural Memory and to select the action best serving its goals (Action Selection).

\footnotetext{
${ }^{3}$ In LIDA, the term codelet refers to small, special purpose processors or running pieces of software code; and corresponds to 'processors' in Global Workspace Theory (Baars \& Franklin, 2009).

${ }^{4}$ We use 'salient' as an umbrella term for percepts which are important, urgent, insistent, novel, threatening, promising, arousing, unexpected, etc.
}

Fig. 1 contains a tentative mapping from spatially relevant modules and mechanisms in LIDA to those in the brain, described below. It is intended to provide a starting point for the implementation of these mechanisms (taking inspiration from the underlying neural correlates), as well as to clarify LIDA's functionality to readers with relevant neuroscience knowledge by pointing out functional correspondences. This tentative mapping is by no means intended to suggest that LIDA implements exact neural mechanisms. Although heavily inspired by and resting on results from cognitive neuroscience and psychology, LIDA is a model of minds, not of brains (Franklin et al., 2012).

\section{Towards real-world capable spatial memory in LIDA}

The following subsections describe computational extensions made to LIDA in order to allow it to encode, store and recall spatial information obtained from real-world environments. Fig. 2 provides an overview of these extensions. Note that some of these, such as the LIDAROS interface and the visual recognition mechanism in EPAM (Extended PAM), do not have correspondents in conceptual LIDA, and are not claimed to plausibly model minds. Rather, they use already existing technologies for solving low-level problems (mainly vision and motor control), which are outside the scope of this work. Although efforts are underway to implement these mechanisms in a cognitively plausible fashion (see e.g. McCall \& Franklin (2013) and Agrawal \& Franklin (2014) for perceptual learning via cortical learning algorithms and Dong \& Franklin (2015a, 2015b) for action execution), they are not yet mature enough to facilitate the present application scenario.

\section{Visual recognition and perceptual representation}

LIDA's PAM contains nodes and links which are the building blocks of 'node structures', which are similar to and inspired by Barsalou's perceptual symbols (Barsalou, 1999; Franklin et al., 2014). PAM nodes represent higher-level features, such as objects, categories, relations, events, situations, and feelings/emotions; and are connected by PAM links, which are weighted and allow passing activation between the nodes. In the implementations in this paper, we have extended LIDA's PAM by an object recognition system based on a convolutional neural network (CNN), yielding EPAM (Extended PAM).

CNNs are a kind of deep learning architecture designed to process $2 \mathrm{D}$ or $3 \mathrm{D}$ data such as images - on which they have led to several breakthroughs (LeCun, Bengio, \& Hinton, 2015) - and are usually trained by a gradient descent procedure called backpropagation. This algorithm has been criticized as not being biologically realistic (Stork, 1989) (although there are versions of deep learning that can be implemented by biological neurons (Bengio, Mesnard, Fischer, Zhang, \& Wu, 2015)). However, despite these arguments concerning implementation, the representations found by state-of-the-art CNNs trained on real-world images are highly similar to those recorded in the inferior temporal (IT) cortex of human and nonhuman primates 


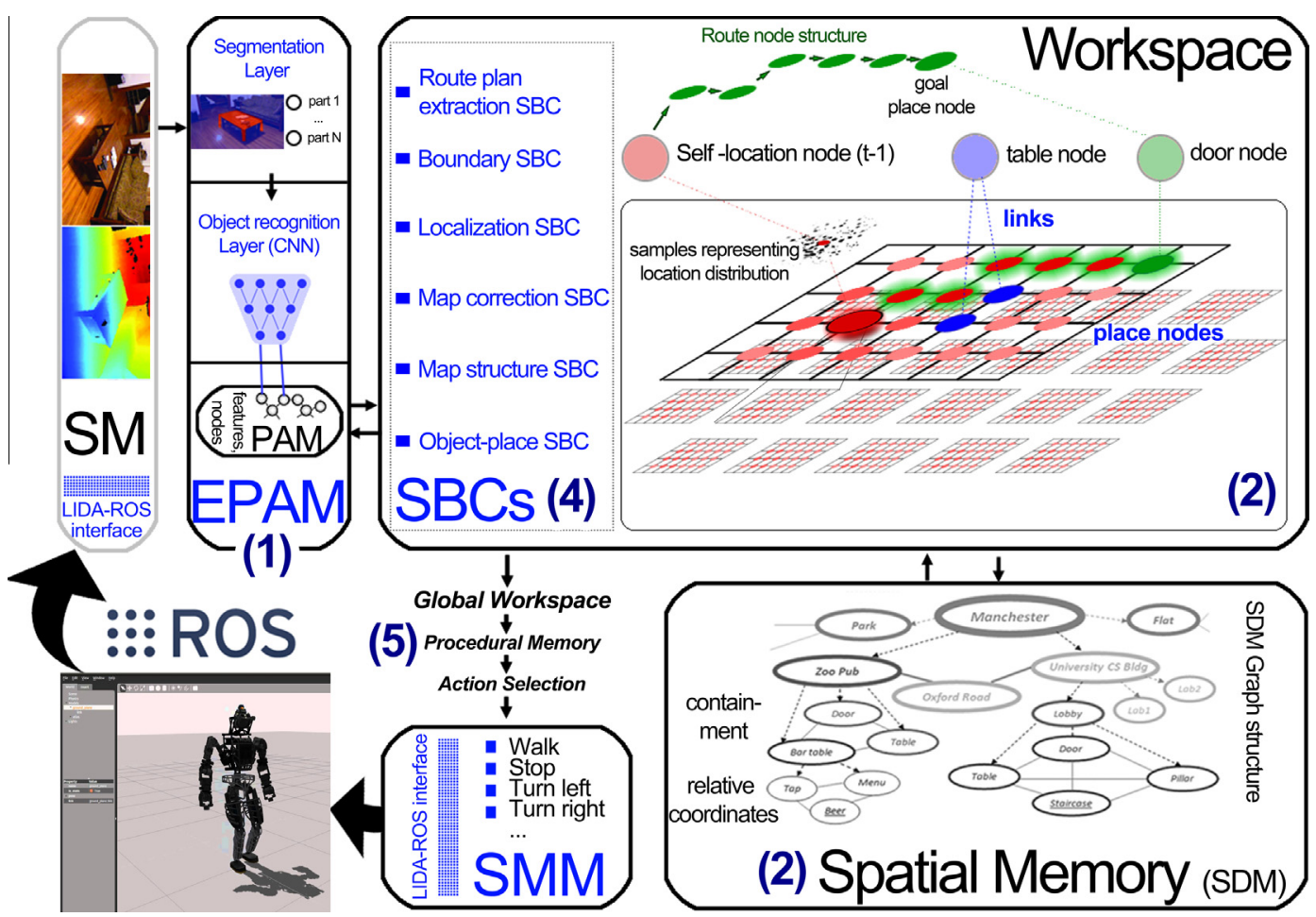

Fig. 2 Extensions to add spatial abilities to LIDA. From the bottom left, clockwise: the LIDA-ROS interface transmits image and depth information (from stereo disparity) from the robot's cameras to Sensory Memory (SM). Object recognition is performed by CNNs in EPAM (Extended PAM), which pass activation to recognized PAM nodes representing objects. These can be associated with place nodes corresponding to their most likely location in the Workspace (determined using the mean of the samples representing their location probability distributions). Place nodes, links between them, and object associations constitute 'cognitive maps', and are constructed, updated, and organized by Structure Building Codelets (SBCs). Place nodes with enough activation to be broadcast consciously can be learned as long-term SDM representations; and can recruit route-following behaviours in Procedural Memory and Action Selection, leading to the execution of a low-level action in Sensory-Motor Memory (SMM), which is transferred to ROS via the LIDA-ROS interface. (Numbers in brackets: see Fig. 1.)

(Khaligh-Razavi \& Kriegeskorte, 2014; Yamins, Hong, Cadieu, \& DiCarlo, 2013).

We have extended PAM with pre-trained $\mathrm{CNNs}^{5}$ for object recognition (Szegedy et al., 2014) and road detection (Brust, Sickert, Simon, Rodner, \& Denzler, 2015) - see Fig. 3. The top layer (softmax layer) of the former was replaced by a classifier trained offline using a dataset of the buildings used in the Gazebo simulation, which was rendered from different perspectives and distances. (Learning should happen in a development fashion in LIDA, not offline; but this exceeds the scope of the current work.) Since CNNs perform best on images containing a single object in the foreground, and have difficulties with clutter, camera images were first segmented, and object recognition performed on the individual segments.

\section{Spatial extensions to LIDA - overview}

As described in Section 'Spatial memory in brains', in brains, hippocampal place cells encode animals' current location in the environment, as well as providing object-

\footnotetext{
${ }^{5}$ These CNNs were available from https://github.com/ BVLC/caffe/wiki/Model-Zoo and https://github.com/cvjena/cn24.
}

place associations. Their equivalent in LIDA is implemented via a special type of PAM nodes, 'place nodes', each of which represent a specific region in the environment, and which reside in the Workspace (as part of the Current Situational Model). Place nodes can be associated with objects perceived to be at that particular location via PAM links for example, agents' self-representation ('self' PAM node) can be associated with the place node representing their most likely location (which needs to be regularly updated). They are also initially connected recurrently to all their neighbours via PAM links. This has been argued to be a plausible connectivity pattern of the hippocampus (Csizmadia \& Muller, 2008; Moser et al., 2008; Samsonovich \& McNaughton, 1997).

Any PAM node in the Workspace representing currently or recently perceived objects (obstacles, landmarks, goals, etc.) in LIDA's Workspace can be associated via PAM links with spatial locations represented by place nodes. A node structure comprised of such object nodes, association links, and place nodes together constitute a 'cognitive map'. Multiple 'cognitive maps' can be used within the same environment in a hierarchical fashion. (There can be maps and sub-maps on different scales and resolutions, and relative position and containment relations between them.) This is 

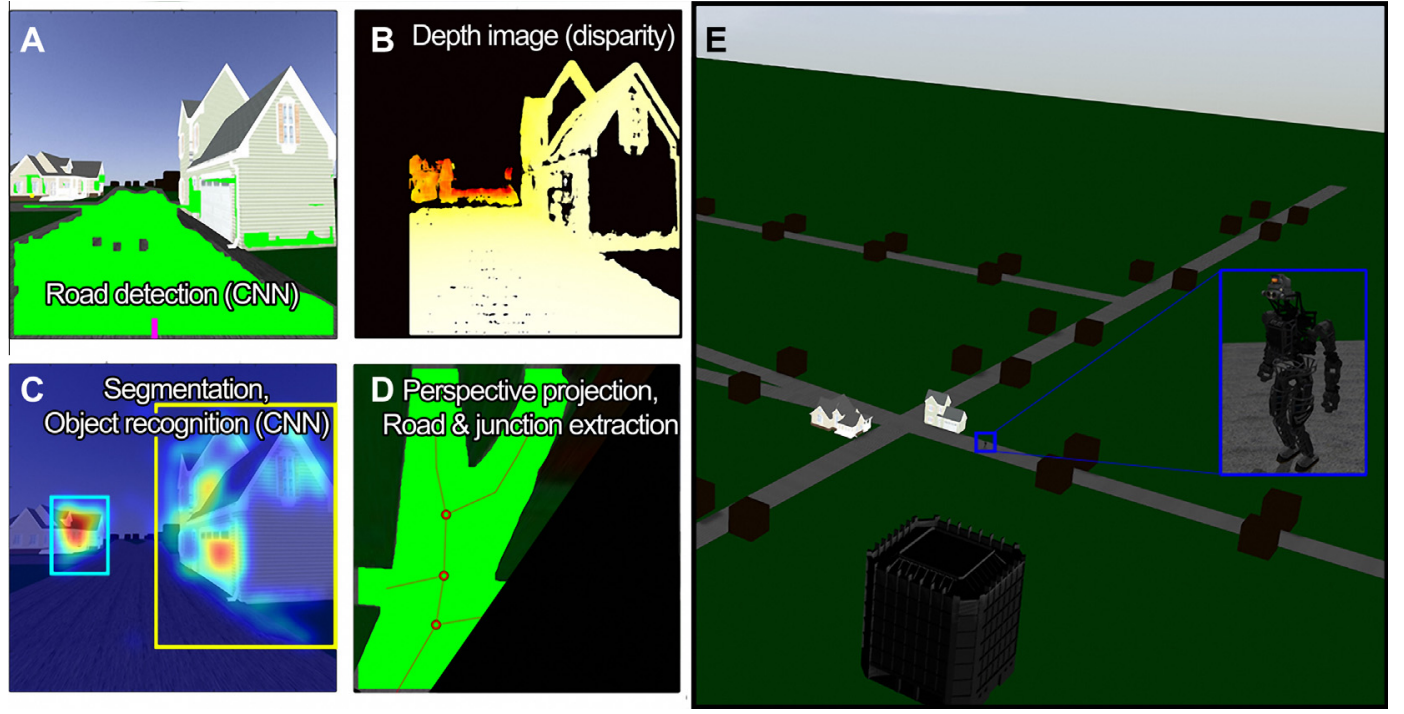

Fig. 3 Representations in Extended PAM (A)-(D) in one of the environments recreated in the Gazebo simulator (E). (A) Camera image with detected road. (B) Depth image from binocular disparity. (C) Likely objects from segmentation (hot colours), recognized by a CNN. (D) Perceived road after denoising and projection based on the depth image.

consistent with neural and behavioural evidence that the human cognitive map is structured (Derdikman \& Moser, 2010) and hierarchical (Hirtle \& Jonides, 1985) (see Madl et al. (submitted for publication) for more extensive literature and evidence). It should be mentioned that the regular grid-like pattern of these place nodes, imposed for computational simplicity, is not biologically realistic, as no regularities have been found in the distribution of firing fields of place cells. (However, a regular grid has been observed in the EC.)

Although these maps are temporary, created and updated in the Workspace, they can be stored in the Spatial Memory module (which can encode trees and sequences (Snaider \& Franklin, 2014)) as long-term memories if they are salient enough to be broadcasted consciously. This long-term memory storage mechanism has not been implemented yet.

Cognitive maps are assembled and updated by structurebuilding codelets (SBC) in the Workspace (LIDA's preconscious working memory). Each of these SBCs addresses a computational challenge associated with endowing an autonomous agent with spatial capabilities (see Fig. 2):

- The 'Object-place SBC' associates large objects recognized by EPAM with place nodes, making use of distance information from stereo disparity to infer their approximate position and size.

- The 'Boundary SBC' detects boundaries in the Workspace, removing links at the locations of these boundaries (currently performed at the boundaries of recognized roads), only leaving links between traversable places (facilitating planning).

- The 'Localization SBC' is responsible for updating the link between the Self PAM node and the place node representing the agents most likely current position in the environment, using Bayesian inference to combine spatial cues.
- The 'Map correction SBC' corrects the map (closes the loop) based on revisited locations (see next section).

- The 'Map structure SBC' spawns new cognitive maps from parts of the current map, based on the proximity of objects represented on a map, in a process resembling clustering; and

- The 'Route plan extraction SBC' extracts shortest routes if a goal representation is present in the Workspace.

\section{Map structuring}

The Map structure SBC processes all place nodes that have associated objects, and clusters these objects based on (1) their spatial location; (2) functional similarity; and (3) the boundaries separating them, using Bayesian nonparametric clustering (as described and substantiated experimentally in Madl et al. (submitted for publication)). Apart from accounting for the structure of cognitive maps, Bayesian nonparametric models have also been successful at accounting for category learning (Sanborn, Griffiths, \& Navarro, 2006) and unifying rational models of categorization (Griffiths, Canini, Sanborn, \& Navarro, 2007). This SBC groups together objects that are close to each other along the given features (in our case, spatial distance and functional similarity). The Map structure SBC spawns a new cognitive map (sub-map) for each identified cluster, consisting of the objects in that cluster and their place nodes; and adjusts the density of place nodes depending on the area of this cognitive map (so that large-scale maps contain a low-resolution and small-scale maps a highresolution place node grid). This process leads to a hierarchy of cognitive maps, a structure suggested to be employed by human spatial memory (Hirtle \& Jonides, 1985; Madl et al., submitted for publication; McNamara, Hardy, \& Hirtle, 1989). 


\section{Localization and mapping}

The Localization SBC is responsible for updating the agents' estimated location after each movement, by linking its Self PAM node with the place node representing this location, as well as for updating landmark locations in a similar fashion. Simply using path integration (odometry) to add up selfmotion signals keeps accumulating errors (Etienne et al., 1996; Jeffery, 2007). This problem has been tackled in robotics in the framework of Bayesian inference, integrating information from odometry with sensory observations in a statistically optimal fashion (Thrun \& Leonard, 2008). It has been argued that brains might employ a similar mechanism (Cheung, Ball, Milford, Wyeth, \& Wiles, 2012; Madl et al., 2014).

Probability distributions representing the location of the agent, as well as the locations of recognized objects, are encoded by means of a set of samples attached to EPAM nodes in the Workspace and manipulated by the Localization SBC (see Fig. 2). There are strong arguments for the neural plausibility of sampling-based uncertainty representations and inference (Fiser, Berkes, Orbán, \& Lengyel, 2010). After every movement, the Localization SBC performs three steps. First, the location estimate of the agent is moved based on the self-motion signal. Second, the self-location estimate and the landmark location estimates are corrected in a Bayesian fashion. Finally, the links of the nodes representing them are updated to the place node corresponding to the best estimate.

These steps correspond to the common implementation of the Kalman filter (Thrun, Burgard, \& Fox, 2005), extended versions of which are still in use for SLAM in robotics, where large matrices are used to keep track of the locations and covariances of all landmarks (requiring the updating of $O\left(N^{2}\right)$ entries at each movement). Another computationally more efficient method (Montemerlo \& Thrun, 2007), with a sampling-based representation of probability distributions, inspired our solution (together with evidence that place cell activity spikes can be seen as samples from a Bayesian posterior (Madl et al., 2014)).

We begin by formalizing path integration, given a motion model $p(\boldsymbol{x} \mid \boldsymbol{m})$ prescribing how the location $\boldsymbol{x}$ changes with a movement $\boldsymbol{m}$ (Table 1 provides a list of all the symbols used in the text). The location at time $t$ can be inferred based on the most recent movement $\boldsymbol{m}_{t-1}$ by integrating out the previous location:

$p\left(\boldsymbol{x}_{t} \mid \boldsymbol{m}_{1: t}\right)=\int p\left(\boldsymbol{x}_{t} \mid \boldsymbol{x}_{t-1}, \boldsymbol{m}_{t-1}\right) \cdot p\left(\boldsymbol{x}_{t-1} \mid \boldsymbol{m}_{1: t-1}\right) \mathrm{d} \boldsymbol{x}_{t-1}$.

Uncorrected, adding up movements like this would incur ever-increasing errors (Etienne et al., 1996). However, we can use Bayes' theorem to calculate the posterior location estimate $\boldsymbol{x}_{t-1}$ of the previous timestep, corrected by observations $\boldsymbol{o}_{1}, \ldots, \boldsymbol{o}_{N} \in O_{t-1}$ of landmark positions $\boldsymbol{l}_{1}, \ldots, \boldsymbol{l}_{N} \in L$ in that timestep (making use of the conditional independence of landmark positions given a location (Montemerlo \& Thrun, 2007)):

$p\left(\boldsymbol{x}_{t-1} \mid O_{t-1}, L_{t-1}, m_{1: t-1}\right)=\gamma p\left(\boldsymbol{x}_{t-1} \mid m_{1: t-1}\right) \cdot \prod_{j=1}^{N} p\left(\boldsymbol{l}_{j} \mid \boldsymbol{o}_{t-1, j}, \boldsymbol{x}_{t-1}\right)$, where $\gamma$ is a normalization constant. We can use this corrected posterior instead of the uncorrected previous path integration estimate $p\left(\boldsymbol{x}_{t-1} \mid \boldsymbol{m}_{1: t-1}\right)$ in Eq. (1), yielding a recursive equation for corrected location estimation (Montemerlo \& Thrun, 2007):

$$
\begin{aligned}
p\left(\boldsymbol{x}_{t} \mid \boldsymbol{m}_{1: t}, O_{1: t}, L\right)= & \gamma \prod_{j=1}^{N} p\left(\boldsymbol{l}_{j} \mid \boldsymbol{o}_{t, j}, \boldsymbol{x}_{t}\right) \\
& \cdot \int p\left(\boldsymbol{x}_{t} \mid \boldsymbol{x}_{t-1}, \boldsymbol{m}_{t-1}\right) p\left(\boldsymbol{x}_{t-1} \mid O_{t-1}, L, m_{1: t-1}\right) \mathrm{d} \boldsymbol{x}_{t-1} .
\end{aligned}
$$

This recursive location estimation equation can be implemented by iterating the three mentioned steps - movement, correction, update - and by using rejection sampling to approximate the statistically optimal posterior in the correction step.

We recently presented evidence that hippocampal place cells are able to perform Bayesian correction, based on neuronal recordings of several hundred place cells and multiple different environments, in which the firing fields of these cells corresponded to the predictions of a Bayesian cue integration model (Madl et al., 2014). In the same paper, we have also suggested how coincidence detection, observed in place cells (Jarsky, Roxin, Kath, \& Spruston, 2005; Katz, Kath, Spruston, \& Hasselmo, 2007; Takahashi \& Magee, 2009), can implement multiplication required to calculate a Bayesian posterior, as well as rejection sampling. The Localization SBC solves Eq. (3) in a manner similar to this coincidence detection mechanism in place cells. The Self-location node keeps track of a number of samples representing the estimated location distribution (however, only the expected value, i.e. the mean of these samples, is connected to a place node and can be broadcast consciously in the model). New samples are generated (and old samples moved) based on the current movement speed $v$ and some Gaussian noise reflecting movement errors whenever the agent moves: $\boldsymbol{s}_{t}^{i}=\overline{\boldsymbol{s}_{t-1}}+T\left(\boldsymbol{v} \Delta t \cdot \mathcal{N}\left(\mathbf{1},\left[\begin{array}{cc}\sigma_{v}^{2} & 0 \\ 0 & \sigma_{\omega}^{2}\end{array}\right]\right)\right)$, where $\sigma_{v}$ and $\sigma_{\omega}$ are linear and angular path integration error parameters, and $T$ transforms from polar (linear and angular speed) to Cartesian coordinates, and $\overline{\boldsymbol{s}_{t-1}}$ is the arithmetic mean of all previous samples $s_{t-1}^{i}$. Note that the self-movement $v \Delta t$ is itself noisy and inaccurate (the multiplicative Gaussian ensures that the samples are spread out enough to likely encompass the unknown true location, if the path integration error parameters are greater than or equal to the actual path integration error).

This movement equation alone would accumulate errors, and spread out samples more and more. To avoid this, it is corrected in a Bayes-optimal fashion by rejection sampling, i.e. by rejecting (discarding) samples $\boldsymbol{s}_{t}^{i}$ inconsistent with current observations. Specifically, samples are retained with a probability proportional to the product of distributions representing currently observed landmarks $\prod_{j=1}^{N} p\left(\boldsymbol{l}_{j} \mid \boldsymbol{o}_{t, j}, \boldsymbol{x}_{t}\right)$ (see Supplementary Information in Madl et al. (2014) for proof that this approximates the Bayesian posterior location). The most likely corrected location can be subsequently obtained from the mean of the remaining samples, $\boldsymbol{x}_{t}=\overline{\boldsymbol{s}_{t}^{\prime}}$. The Localization SBC then updates a link 
Table 1 Mathematical symbols used in the text.

\begin{tabular}{ll}
\hline Symbol & Description \\
\hline $\boldsymbol{x}_{t}$ & Location in 2D space at time $t$ \\
$\boldsymbol{m}_{t}$ & Motion vector in 2D space at time $t$, based on motor command \\
$\boldsymbol{o}_{i}$ & Observed distance of landmark $i$ \\
$\boldsymbol{O}_{t}$ & Observed distances of all landmarks at time $t: O_{t}=\left\{\boldsymbol{o}_{t, 1}, \ldots, \boldsymbol{o}_{t, n}\right\}$ \\
$\boldsymbol{l}_{i}$ & Location of landmark $i$ in 2D space \\
$L_{t}$ & Locations of all landmarks at time $t: L_{t}=\left\{\boldsymbol{I}_{t, 1}, \ldots, \boldsymbol{I}_{t, n}\right\}$ \\
$\boldsymbol{v}$ & Movement speed \\
$\mathcal{N}(\boldsymbol{\mu}, S)$ & Normal (Gaussian) distribution with mean $\mu$ and covariance $S$ \\
$\boldsymbol{s}_{t}^{i}$ & Sample $i$ from the location distribution at time $t$ \\
$\boldsymbol{s}_{t}$ & Mean of all samples from the location distribution \\
$\boldsymbol{c}_{i}$ & Constraint $i$ specifying the measured distance of two locations $\boldsymbol{x}_{a}$ and $\boldsymbol{x}_{b}($ e.g. from path integration, \\
$\boldsymbol{d}_{i}$ & or recognized revisited places) \\
$S_{i}$ & Discrepancy between constraint $i$ and the corresponding estimated locations: $\boldsymbol{d}_{i}=\mathbf{x}_{a}-\boldsymbol{x}_{b}-\boldsymbol{c}_{i}$ \\
$\boldsymbol{C}$ & Covariance matrix expressing the uncertainty associated with a constraint \\
$\boldsymbol{X}$ & All constraints (measurements) acquired in the current loop: $C=\left\{\boldsymbol{c}_{1}, \ldots, \boldsymbol{c}_{n}\right\}$ \\
$\gamma$ & All recent locations (entire path) estimated in the current loop: $X=\left\{\boldsymbol{x}_{1}, \ldots, \boldsymbol{x}_{m}\right\}$ \\
$J$ & Normalization constant \\
\hline
\end{tabular}

between the Self-location node and the correct place node corresponding to $\boldsymbol{x}_{t}$.

Analogously, the same rejection sampling mechanism can also be used to keep track of most likely landmark locations, implementing:

$p\left(\boldsymbol{l}_{j} \mid \boldsymbol{o}_{t, j}, \boldsymbol{x}_{t}\right)=\gamma p\left(\boldsymbol{o}_{t, j} \mid \boldsymbol{l}_{j}, \boldsymbol{x}_{t}\right) p\left(\boldsymbol{l}_{j} \mid \boldsymbol{o}_{t-1, j}, \boldsymbol{x}_{t-1}\right)$,

under the assumption that the data association problem (the question which landmark the measurements $\boldsymbol{o}$ belong to) can be solved accurately using the CNN described above.

The movement step (path integration) has been shown to be performed by grid cells in the entorhinal cortex (McNaughton et al., 2006), and the correction step by place cells (Madl et al., 2014). Finally, we have argued that phase resetting observed in grid cells can implement the update step, completing the localization cycle. Based on the observation that it partially accounts for single-cell recording data in multiple environments (Madl et al., 2014) and that it can be implemented as a biological neural network in a straightforward fashion, we think this kind of Bayesian correction constitutes a plausible model of local spatial error correction (see Fig. 4).

\section{Route planning}

The Route plan extraction SBC creates PAM node structures representing the shortest path to the agent's current goal if such a goal is currently present in the Workspace. If there are multiple cognitive maps present in the Workspace, it selects the highest-resolution map containing both start and goal location. Such maps are implemented as recurrently interconnected place node networks, which facilitate a very simple path planning mechanism (Fig. 5). Assuming that every goal location $G$ passes activation through the network, the distance to the goal can be decreased by moving to the adjacent neighbour node with the highest activation. If the nodes representing the locations of possible obstacles are connected with zero or near-zero weights, this mecha- nism can implement obstacle avoidance as well as path planning. Crucially, this activation-based planning mechanism operates on a hierarchy of 'cognitive maps' rather than on a single level. We argue that this allows better solutions of multi-goal navigation problems such as the travelling salesman problem. The evaluation of this planning mechanism against human data was briefly described in Madl et al. (2013) (for details see the Supplementary Information).

\section{Loop closing - fixing previously learned maps}

If uncorrected, accumulating path integration errors eventually render learned spatial representations useless - a problem necessitating the use of other modalities for map learning. Integrating spatial information in an approximately statistically optimal (Bayesian) fashion, as described above, helps correct local maps. However, only the agent's current location and the locations of currently perceived objects are updated with our procedure. When traversing large cycles (loops) in an environment and returning to a previously visited location, the remaining errors still accumulate and prevent this loop from being represented correctly, causing multiple representations of the same places (of subsequently revisited places) - see Fig. 6.

Therefore, a mechanism is needed to correct the representation of locations encountered during loops (such a correction is called 'loop closure' or 'closing the loop' in the robotics literature (Williams et al., 2009)). This section outlines a biologically plausible solution to this problem, as well as its relation to phenomena observed in hippocampal neurons. This solution is also used by the Map correction SBC to correct errors in learned cognitive maps.

Although the problem of accumulating errors and the resulting need to correct maps with sensory information has been identified early in spatial modelling literature (McNaughton et al., 1996), the question how brains might 'close the loop' has received very little attention, and no 


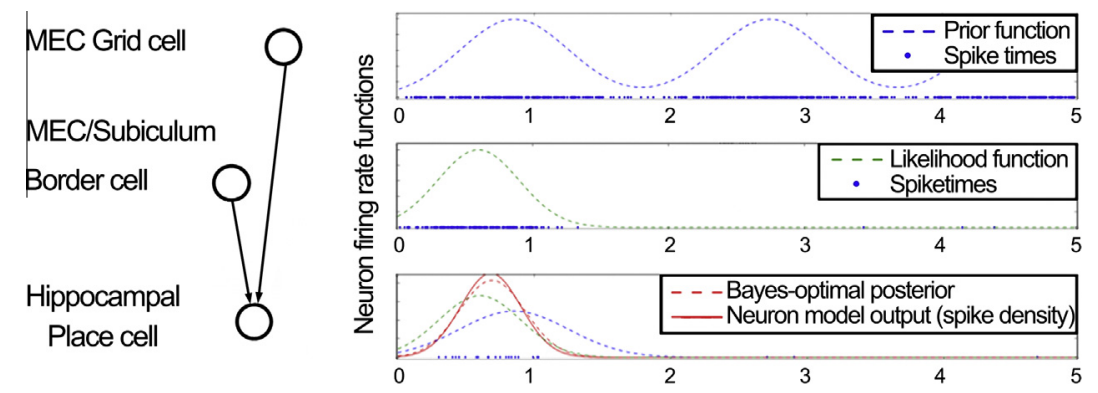

Fig. 4 Approximate Bayesian cue integration in spiking neurons. Calculating the posterior probability distribution of the current location (Eq. (2)) requires multiplying a prior location distribution from path integration (represented by grid cells) with likelihood distributions from measurements of objects or boundaries (here represented by a border cell). Each spike can be seen as a sample from a probability distribution. If the place cell receiving input from the grid and border cells performs coincidence detection, which can be seen as approximate multiplication or rejection sampling (Madl et al., 2014), yielding an approximate Bayesian posterior, and representing the associated uncertainty via the size of its firing field. A Bayesian model can account for hippocampal place field sizes in behaving rats. Figure adapted from Madl et al. (2014).

A

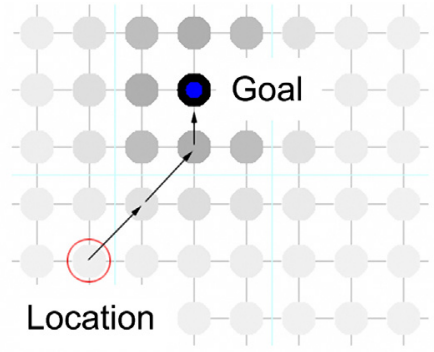

C

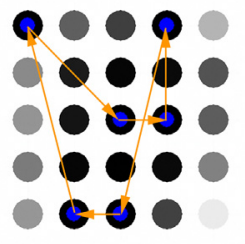

B

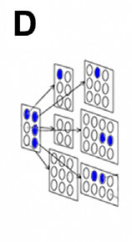

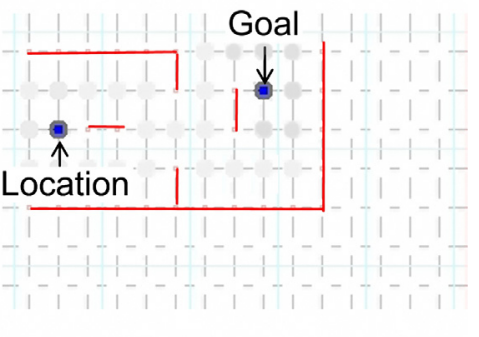

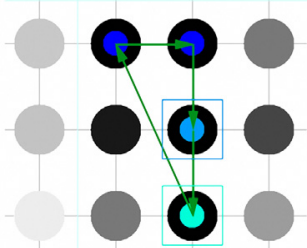

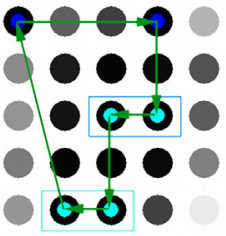

Fig. 5 Route planning on recurrently interconnected place nodes. (A) Single goal routes can be obtained by following an activation gradient to a goal. (B) Obstacle avoidance can be implemented by setting connection weights to zero near boundaries (red lines). (C) On a flat grid, following activation gradients can lead to sub-optimal paths for multi-goal navigation. (D) However, when operating on a hierarchy - planning rough, low-resolution routes first, and then refining them on higher resolution maps - this mechanism can yield near-optimal solutions.

plausible mechanisms have been proposed to the authors' knowledge. The large majority of robotics solutions to this problem require many iterations over huge matrices containing information regarding every position ever visited (Bailey \& Durrant-Whyte, 2006; Durrant-Whyte \& Bailey, 2006; Thrun \& Leonard, 2008; Williams et al., 2009), and they are thus neurally implausible. However, a probabilistic perspective on this problem can still help find a plausible candidate algorithm, consistent with hippocampal replay as the correction mechanism, which we summarize below.

First, let us assume that it is sufficient to correct the route taken during the loop. Local, currently perceived landmark positions are corrected separately as described above. When performing large-scale loop closing, our scheme applies the same correction to a position and the local landmarks around it. ${ }^{6}$ We also make the assumption that correction only concerns position representations and not angular representations, since there is neuronal evidence for the former but not the latter (replay of encountered information happens in place cells, but has not been observed for direction-sensitive neurons such as headdirection cells in the postsubiculum (Brandon, Bogaard, Andrews, \& Hasselmo, 2012)).

The available information includes the path $X$ consisting of estimated, recently visited locations $\boldsymbol{x}_{0}, \ldots, \boldsymbol{x}_{m} \in X$, and

\footnotetext{
${ }^{6}$ Unlike the strong evidence for hippocampal replay concerning place cells representing recently visited locations, it is unclear whether cells associated with landmarks are also 'replayed'. Therefore, we forego separate landmark correction in loops for now.
} 


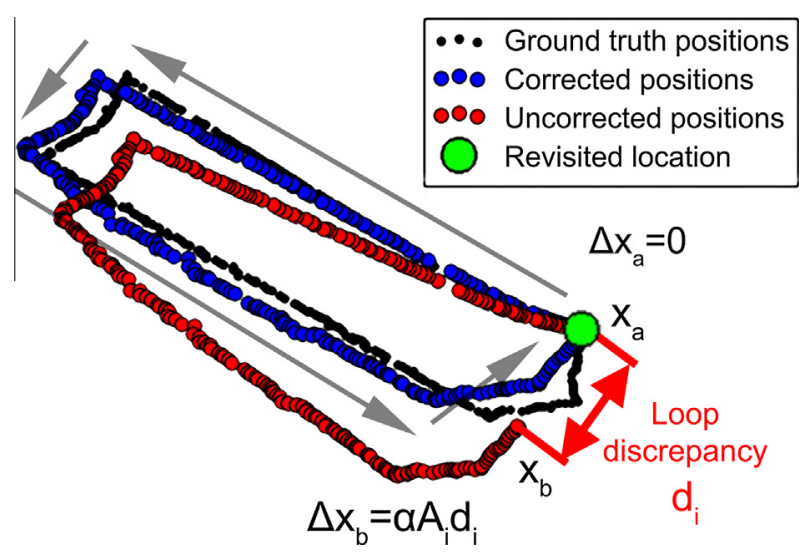

Fig. 6 Loop closing performed by the Map correction SBC. Correcting estimated positions along a path when re-visiting a known place (large green dot), after traversing a large loop. Recognizing this place yields the knowledge that current estimated location $\boldsymbol{x}_{b}$ should equal $\boldsymbol{x}_{a}$; and the correction $\boldsymbol{d}_{i}$ based on the discrepancy is applied proportionally to all visited places along the loop. This backward correction is consistent with hippocampal replay.

a set of constraints $\boldsymbol{c}_{1}, \ldots \boldsymbol{c}_{m} \in C$ specifying how far two locations should be from each other - this includes distances from the path integration system for subsequent locations, and equivalence constraints (with zero distance) when revisited places are recognized. We will temporarily assume simultaneous access to all path integration constraints, and will drop this implausible requirement later. Each constraint between two locations is represented as a Gaussian with the measured distance $c_{i}$ as the mean, and the associated uncertainty represented by the covariance $S_{i}$ (e.g. path integration is inexact - high uncertainty; but a recognized revisited place is at the same location - low uncertainty). The correct path is the one that is most consistent with all known constraints (known distances between the locations); or, from a probabilistic perspective, the one that maximizes the conditional probability of the locations constituting the path, given the constraints ${ }^{7}$ :

$P(X \mid C) \propto \prod_{i=1}^{m} P\left(c_{i} \mid X\right)$.

Since each constraint is represented as a Gaussian over the distance between a pair of locations $a_{i}$ and $b_{i}$, $P\left(\boldsymbol{c}_{i} \mid X\right) \propto \mathcal{N}\left(\boldsymbol{x}_{a}-\boldsymbol{x}_{b} ; \boldsymbol{c}_{i}, S_{i}\right)$, and the conditional probability is

$P(X \mid C) \propto \prod_{i=1}^{m} \exp -\left(\frac{1}{2}\left\|x_{a}-x_{b}-c_{i}\right\|_{S_{i}}\right)$.

We will denote the discrepancy between the constraint $i$ and the difference between corrected locations $a_{i}$ and $b_{i}$ as $\boldsymbol{d}_{i}=\boldsymbol{x}_{a}-\boldsymbol{x}_{b}-\boldsymbol{c}_{i}$. Under ideal conditions without noise and

\footnotetext{
${ }^{7}$ In robotic SLAM solutions, the path likelihood would also depend on all landmark observations. We omit them here because our loop closing procedure updates each position along with the path together with its local landmarks, applying the same translation to both, which renders the observation conditionals constant; once again sacrificing accuracy for plausibility.
}

errors, all $d_{i}$ would be zero; but in realistic environment there will be discrepancies between estimated and measured differences. The 'best' path estimate maximizes $P(X \mid C)$, or equivalently minimizes its negative logarithm $-\log P(X \mid C)$ (minimizes the discrepancies):

$X_{M L}=\underset{X}{\arg \max } P(X \mid C)=\underset{X}{\arg \min } \sum_{i=1}^{m}\left\|\boldsymbol{d}_{i}\right\|_{S_{i}^{-1}}$

Eq. (7) can be written in matrix form and solved via Gauss-Seidel iteration, in a way that only requires a few alternating forward and backward passes over the path (see Supplementary Information); however, alternating replay has not been observed in the hippocampus.

Fortunately, there is a more plausible solution which can be implemented neurally. It has been argued that SpikeTime Dependent Plasticity (STDP) can implement gradient descent in biological neurons (Bengio et al., 2015; Bengio, Mesnard, et al., 2015). Our starting point is the stochastic gradient descent-based maximization of $P(X \mid C)$ described in (Olson, Leonard, \& Teller, 2006), which suggests the following gradient with respect to constraint $i$ :

$\Delta X \approx \alpha\left(J S^{-1} J\right)^{-1} J_{i}^{T} S_{i}^{-1} \boldsymbol{d}_{i}$,

where $\alpha$ is a learning rate, $J$ is the full Jacobian of all constraints with respect to the locations, and $J_{i}$ the Jacobian of constraint $i$. Because constraints apply to locations incrementally (with zero sensory errors, the correct current location would be $\boldsymbol{x}_{c}=\sum_{i} \boldsymbol{c}_{i}$ ), the Jacobian is also incremental, spreading out the discrepancy $\boldsymbol{d}_{i}=\left(\boldsymbol{x}_{a}-\boldsymbol{x}_{b}-\boldsymbol{c}_{i}\right)$ over an entire loop (by means of having a structure similar to the incidence matrix). This means the Jacobian needs not be explicitly computed or represented. For a given loop closed by $c_{i}$ with uncertainty $S_{i}$, let us assume unchanging path integration uncertainties $S_{p}$ for each movement within the loop, and introduce a loop precision parameter $A_{i}$ specifying the uncertainty of the current loop closure in relation to that of path integration, $A_{i}=S_{i} / S_{P}$. The correction applied to any single location $\boldsymbol{x}_{j}$ visited after the recognized previous location $\boldsymbol{a}_{i}$ (i.e. if $j>a_{i}$ ) thus becomes:

$\Delta \boldsymbol{x}_{j} \approx \alpha \boldsymbol{d}_{i} \frac{\sum_{k=a+1}^{j} S_{i}^{-1}}{\sum_{k=a+1}^{\min (j, b)} S_{P}^{-1}}=\alpha A_{i} \boldsymbol{d}_{i} p_{j}$,

where $p_{j}=\left(\min \left(j, b_{i}\right)-a_{i}-1\right) /\left(b_{i}-a_{i}-1\right)$ denotes how far $\boldsymbol{x}_{j}$ lies along the loop, with $0 \leqslant p_{j} \leqslant 1$.

Conveniently, we can neglect path integration constraints - they are already included in the path $X$, and, since they concern subsequent locations with $b=a+1$, they lead to $\Delta x=0$ according to Eq. (9). The updates only concern loop closing constraints. Given that the distance to the same place when re-visiting it is zero, $\boldsymbol{d}_{i}=\boldsymbol{x}_{a}-\boldsymbol{x}_{b}$. Furthermore, we don't have to re-activate all locations ever visited; only those in the loop. The ensuing correction mechanism is simple (and easily implementable with neurons): when a loop closure is detected, the locations along the loop are iteratively corrected with the discrepancy between estimated and observed location according to Eq. (9). The iteration proceeds backwards, starting at the estimated location at the re-visited place, and has to run several times to approximate a near-optimal solution. This is consistent with backward replay of visited locations in 
hippocampal place cells (Carr et al., 2011), with the presence of distances between locations encoded in such replays (Diba \& Buzsáki, 2007), and with the observation that replay happens significantly more often than the number of times the animal re-visits places.

The described procedure is carried out regularly by the Map correction SBC after a loop closure has been detected (when recognizing an already encountered landmark via the CNN in EPAM). It simply spreads out the discrepancy $\boldsymbol{d}_{i}$ proportionally along the place nodes representing the traversed loop, according to Eq. (9) (see Fig. 6). The Map correction SBC also corrects the positions of encountered buildings, and of the traversed road, stored on the cognitive map (i.e., the same correction is applied to building nodes and road nodes as to the $\boldsymbol{x}_{j}$ closest to them). The location of a part of a road or that of a building is corrected by linking the node representing it with the correct place node.

Apart from behavioural predictions regarding cognitive map accuracy, validated in the next sub-section, and the prediction that hippocampal replay (Carr et al., 2011) might (also) serve the purpose of correcting cognitive maps, this suggested mechanism also yields a quantitative prediction on a cellular level, assuming that the synaptic strength place cells depends on the distance $\boldsymbol{d}_{p f}$ between their place fields. For example, Csizmadia and Muller (2008) suggest that the synaptic weight converges to $S=\exp \left(-k d_{p f}\right)$. which for small $k d_{p f}$ can be approximated by $S=1-k d_{p f}$. Furthermore, STDP implies a weight change proportional to the change in post-synaptic voltage potential (Bengio, Mesnard, et al., 2015). Under these assumptions, our suggested cognitive map correction mechanism implies that after re-visiting a location, during subsequent hippocampal replay, for a pair of place cells which are sufficiently close together for the approximation to hold, changes in postsynaptic voltage potential will be approximately proportional to the correction magnitude $\Delta x$, i.e. to the amount the place field has shifted during replay. It is clear from empirical data that place fields shift after re-visiting locations in an environment (Mehta, Quirk, \& Wilson, 2000), and that backward replay contains distance information between place fields (Diba \& Buzsáki, 2007). We leave the verification of the mentioned prediction for future work.

\section{Results}

This section reports results obtained by LIDA agents with the extensions described above, reproducing data from psychological experiments. These experiments were chosen to compare the agent's spatial estimation accuracies, and cognitive map structures, with those of human subjects.

Instead of free exploration, the routes in the experiments below were pre-programmed into the agents' longterm memory, by storing the turns to be taken in the form of schemes (percept-action mappings) in Procedural Memory, for the following reasons. In Experiment 1, closely reproducing the participant trajectories (as opposed to exploration behaviour) was crucial to modelling accumulating uncertainty. In Experiments 2 and 3, subjects' exploration trajectories in their hometowns were not known (having happened years or decades before the experiment).
Furthermore, exploring environments on the scale of the participant cities modelled in Experiment 2 in tractable timeframes would have required an intelligent exploration strategy, which we have not implemented yet in LIDA. Therefore, the agent was given the turns it should take.

All other information came from noisy sensors, and no ground truth information was provided to the agents, which makes the experiments suitable for evaluating spatial representation accuracy.

\section{Experiment 1 - Localization and cue integration}

In order to substantiate the Bayesian localization and cue integration mechanism, we have replicated a behavioural experiment (Nardini, Jones, Bedford, \& Braddick, 2008) investigating the integration of self-motion and sensory information in location estimation. In this experiment, subjects were asked to pick up three glowing objects in a dark room (see Fig. 7B) and, subsequently, to return the first object to its original location. In the self-motion + landmarks condition, there were three landmarks available for orientation, and subjects were not disoriented - both sources of information were available. In the landmarks condition, subjects were disoriented by turning in order to deprive them of orientation information. In the selfmotion condition, subjects were not disoriented, but the glowing landmarks were turned off and were not perceivable in the dark.

To simulate this experiment, the same environmental layout (with accurate object distances) was reproduced in a simulation. The agent went through the same procedure as the participants, and performed Bayesian localization after every movement, as described above. The noise parameters affecting the agent's measurements were set as follows. Distance estimation inaccuracies were set to $3 \%$, which is a frequently observed distance estimation error in virtual (Murgia \& Sharkey, 2009; Waller, 1999) and real environments (Grechkin, Nguyen, Plumert, Cremer, \& Kearney, 2010; Plumert, Kearney, Cremer, \& Recker, 2005). The two remaining noise parameters (linear and angular self-motion estimation inaccuracies) were adjusted to fit the data using coordinate descent. Path integration errors were modelled by multiplicative 1-mean Gaussian noise, since variability in human odometry is proportional to magnitude (Durgin, Akagi, Gallistel, \& Haiken, 2009). The agent was allowed both path integration and landmark information in the first, only landmark information in the second, and only self-motion information in the third condition. Fig. 7 shows the simulation results, which are consistent with the empirical data for adult subjects.

Nardini et al. (2008) point out that adults can integrate spatial cues in a nearly statistically optimal fashion, in accordance with earlier animal studies indicating Bayesian spatial cue integration in brains (Cheng et al., 2007). Thus, the good fit of the Bayesian localization model described above with the human data in Fig. 7 is not surprising. The observation that not only the magnitude of the errors but also the response variances (Fig. 7C) are similar for humans and the model lends credence to our particular implementation of Bayesian localization (using rejection sampling). 

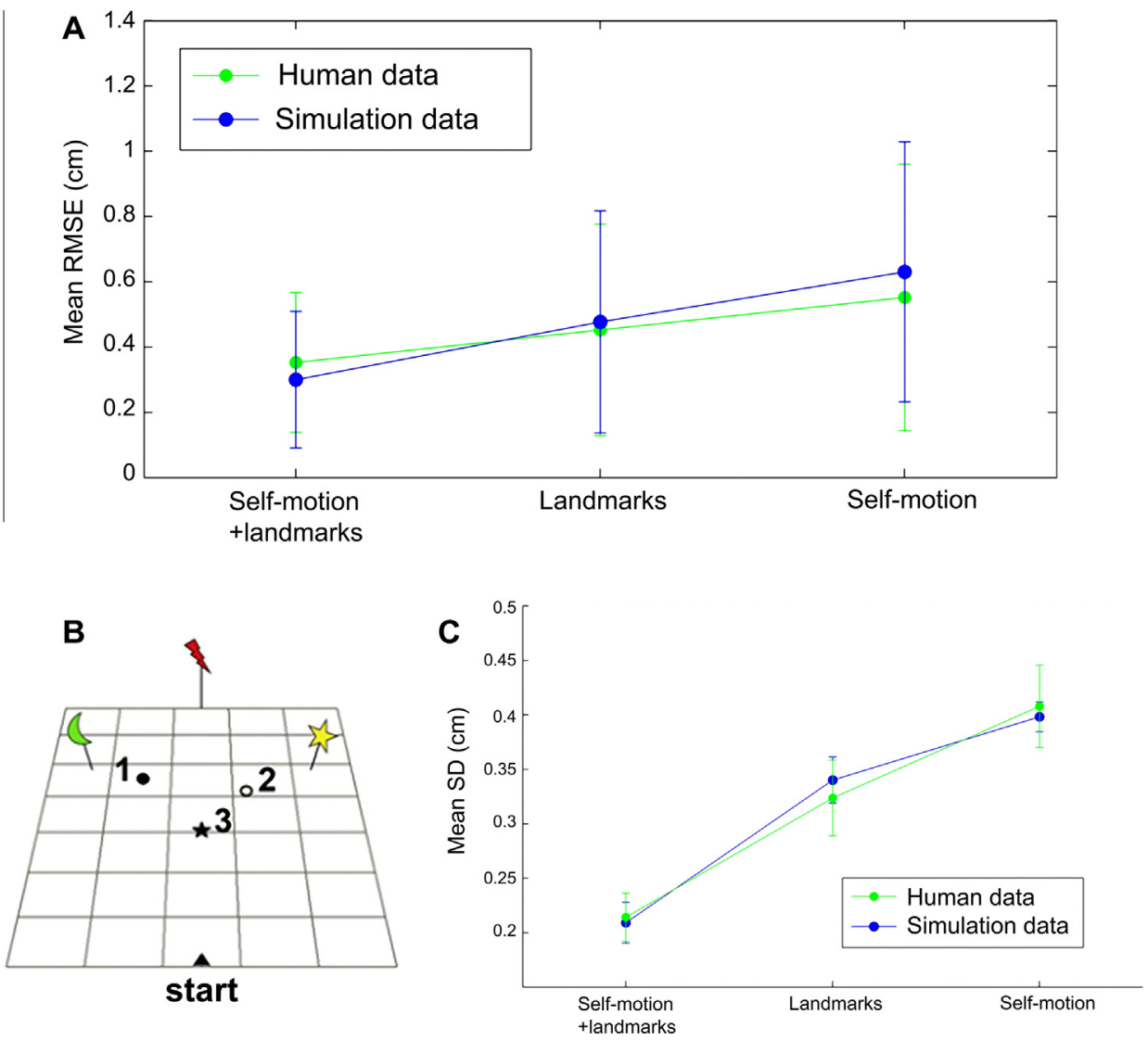

Fig. 7 Position errors and standard deviations in the cue integration experiment by Nardini et al. (2008). (A) Mean RMSE (root mean squared errors) of participants, and mean SD (standard deviation), for the responses of human subjects (green) and the agent (blue), respectively. (B) The experiment environment. Participants had to pick up objects 1-3 in order, and then replace object 1. The colored objects (moon, star, lightning) are the landmarks (from Nardini et al. (2008)). (C) Mean SD of participants (green) and the agents (blue).

However, the present model is unable to account for the behaviour of children younger than eight years of age, who seem to alternate between self-motion and landmark cues, instead of integrating them. A model of the development of cue integration is outside the scope of the present paper.

\section{Experiment 2 - Cognitive map accuracy (real environments)}

Here we replicate map accuracies of Experiment 3B in Madl et al. (submitted for publication), ${ }^{8}$ in which participants were asked to pick 8 very familiar buildings in cities they knew well, within walking distance (such that they knew how to walk from any one to the other). They were then asked to create a sketch map, by indicating the relative positions of these buildings on a featureless canvas on a computer. Sketch maps were linearly translated, rotated and scaled to fit the correct map best using Procrustes analysis (Gower, 1975). Each subject produced three sketch maps, of which those not significantly better than random

\footnotetext{
${ }^{8}$ A pre-print of Madl et al. (submitted for publication) is available
} at http://madlnet.net/tamas/mapstructure.pdf. guessing were excluded. Sketch maps spanning an area larger than $4 \mathrm{~km}^{2}$ were also excluded to reduce computational load. This left 19 participants and a total of 28 different maps (environments) in 21 cities (maps in the same cities concerned different regions).

To reduce computational load, only the roads (and adjacent buildings) were recreated in the simulation, which allowed getting from one of these buildings to the other, i.e., which lay along one of the $\left(\begin{array}{l}8 \\ 2\end{array}\right)=28$ shortest routes between two respective buildings for each map. These roads and buildings were placed at positions with correct real-world distances in the simulation (geospatial information was obtained via Google Maps $\mathrm{API}^{9}$ ), yielding multiple routes several kilometers long.

Within these simulated environments, the agent was initially placed at a randomly selected building, and subsequently traversed the shortest path visiting all buildings. (For reasons described at the beginning of this section, the correct turns were provided to the agent in advance.) Each movement incurred linear and angular path integration errors, which once again were adjusted to fit the data. Each recognized building was stored on the agent's

\footnotetext{
${ }^{9}$ https://developers.google.com/maps/.
} 

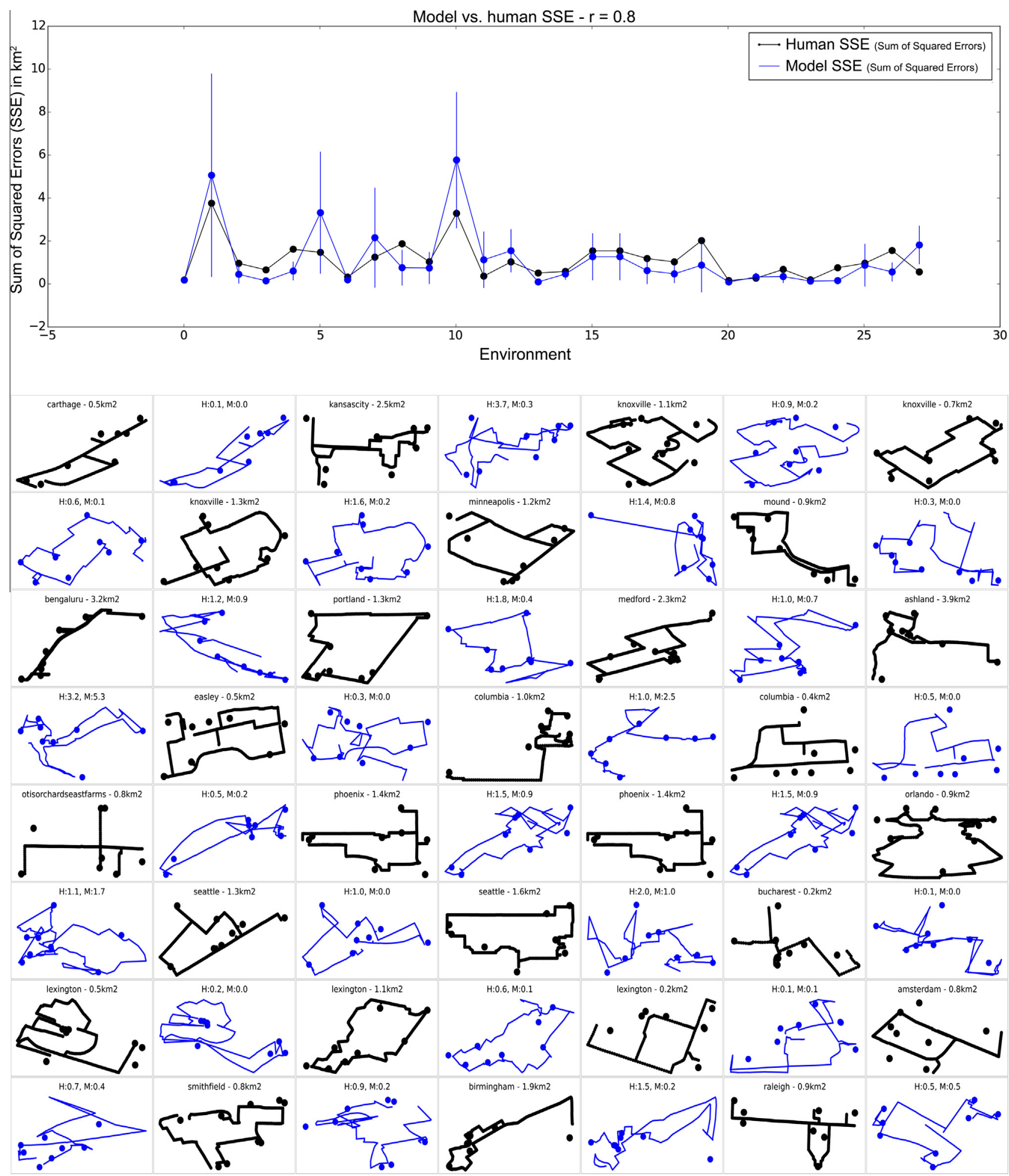

Fig. 8 Comparison with human and model errors over all environments (top), and plots containing the ground truth (black) and learned (blue) street and salient building locations. Titles indicate the city name and region area for the ground truth, and $(\mathrm{H})$ uman and $(M)$ odel errors for the model subplots. Human data from Madl et al. (submitted for publication).

cognitive map, and the map was corrected upon returning to the starting building (loop closure) as described in Section 'Loop closing - fixing previously learned maps', yielding the final maps plotted in Fig. 8 (bottom). Agents traversed each environment 12 times in total, and the errors between the learned building positions and the correct map were averaged for comparison with the human map errors. 
Fig. 8 compares the errors of the maps learned by the agent with human sketch maps, after adjustment of the linear and angular path integration noise parameters by coordinate descent. Map errors are measured as the sum of squared errors (SSE) between the correct geographical building locations, and the locations estimated by the participants/by the model. Unlike the model predictions, which are already in the correct reference frame, human data is linearly translated, rotated and scaled first to fit the correct map. The errors averaged over all maps are $1.07 \mathrm{~km}^{2}$ $(\sigma=0.85)$ for humans, and $1.08 \mathrm{~km}^{2}(\sigma=1.39)$ for the model, and the model errors correlate with human errors with $r_{m, h}=0.80\left(p=2.42 * 10^{-7}\right)$, with a coefficient of determination (proportion of explained variance) of $R^{2}=0.60$ which suggests that the model explains the majority of the variance in human map error data.

The similar ratio of errors between smaller or simpler environments (e.g. Carthage, environment 0 ) and larger or more complex environments (e.g. Kansas City, environment 1) substantiates the plausibility of the proposed mechanism. The biggest shortcoming of the described experimental setup is that the agent always explored the environment along the shortest route connecting the buildings, whereas the human participants may have taken different routes (they explored their respective cities many years ago, and were unable to recall the exact initial routes). Future work will be required to compare learned cognitive map errors in settings with equivalent routes (e.g. by asking subjects to memorize a novel environment, recording their exact routes, and using them for more accurate replication).

Note that this model only uses the eight buildings the participant indicated as being very familiar, in order to recognize having revisited a place and to correct maps. Along routes of this size, humans can presumably re-identify more than these eight places. Even in areas without salient landmarks, a matching visual sequence while walking can trigger a feeling of familiarity. Furthermore, declarative memories (e.g. knowing facts regarding modern city layouts) may help infer and constrain spatial knowledge and correct representations (e.g. planned cities are unlikely to have irregularly shaped roads). We will implement episodic sequencebased place recognition and interactions with declarative memory in future work.

\section{Conclusion}

In order to tackle challenges posed by noisy sensors and complex, uncertain environments, we have extended LIDA by CNN-based perception, and by mechanisms for learning and correcting cognitive maps facilitating navigation. These include novel reinterpretations of coincidence detection in place cells as approximate Bayesian cue integration, and hippocampal replay as cognitive map correction; and suggested computational and algorithmic models of these phenomena, consistent with the 'Bayesian brain' paradigm (Knill \& Pouget, 2004). We have also compared spatial representation accuracies to human subjects. Although a large number of issues remain to be solved for real-world-capable autonomous agents (including developmental learning of perceptual representations and affordances, visual place recognition, long-term spatial and episodic memories, transferring learned spatial knowledge and expectations between environments, and spatial reasoning, to name just a few), we believe these extensions provide a first step towards a cognitive architecture combining biological plausibility and real-world functionality.

\section{Acknowledgements}

This work has been supported by EPSRC (Engineering and Physical Sciences Research Council) Grant EP/1028099/1, and FWF (Austrian Science Fund) Grant P25380-N23.

\section{Appendix A. Supplementary material}

Supplementary data associated with this article can be found, in the online version, at http://dx.doi.org/10. 1016/j.bica.2016.02.001.

\section{References}

Agrawal, P., \& Franklin, S. (2014). Multi-layer cortical learning algorithms. In 2014 IEEE symposium on computational intelligence, cognitive algorithms, mind, and brain (CCMB) (pp. 141-147). IEEE.

Baars, B. J. (2002). The conscious access hypothesis: Origins and recent evidence. Trends in Cognitive Sciences, 6, 47-52.

Baars, B. J., \& Franklin, S. (2009). Consciousness is computational: The LIDA model of global workspace theory. International Journal of Machine Consciousness, 1, 23-32.

Baars, B. J., Franklin, S., \& Ramsoy, T. Z. (2013). Global workspace dynamics: Cortical 'binding and propagation' enables conscious contents. Frontiers in Psychology, 4.

Bailey, T., \& Durrant-Whyte, H. (2006). Simultaneous localization and mapping (slam): Part ii. IEEE Robotics \& Automation Magazine, 13, 108-117.

Barrera, A., Cáceres, A., Weitzenfeld, A., \& Ramirez-Amaya, V. (2011). Comparative experimental studies on spatial memory and learning in rats and robots. Journal of Intelligent \& Robotic Systems, 63, 361-397.

Barry, C., Lever, C., Hayman, R., Hartley, T., Burton, S., O’Keefe, J., ... Burgess, N. (2006). The boundary vector cell model of place cell firing and spatial memory. Reviews in the Neurosciences, 17, 71-97.

Barsalou, L. W. (1999). Perceptual symbol systems. Behavioral and Brain Sciences, 22, 577-660.

Bengio, Y., Lee, D. H., Bornschein, J., \& Lin, Z. (2015). An objective function for stdp. Available from 1509.05936.

Bengio, Y., Mesnard, T., Fischer, A., Zhang, S., \& Wu, Y. (2015). Towards biologically plausible deep learning. Available from 1502.04156.

Bird, C. M., \& Burgess, N. (2008). The hippocampus and memory: Insights from spatial processing. Nature Reviews Neuroscience, 9, 182-194.

Brandon, M. P., Bogaard, A. R., Andrews, C. M., \& Hasselmo, M. E. (2012). Head direction cells in the postsubiculum do not show replay of prior waking sequences during sleep. Hippocampus, 22, 604-618.

Brust, C. A., Sickert, S., Simon, M., Rodner, E., \& Denzler, J. (2015). Convolutional patch networks with spatial prior for road 
detection and urban scene understanding. Available from 1502. 06344.

Burgess, N. (2008). Spatial cognition and the brain. Annals of the New York Academy of Sciences, 1124, 77-97. http://dx.doi. org/10.1196/annals.1440.002.

Burgess, N., Jackson, A., Hartley, T., \& O’keefe, J. (2000). Predictions derived from modelling the hippocampal role in navigation. Biological Cybernetics, 83, 301-312.

Carr, M. F., Jadhav, S. P., \& Frank, L. M. (2011). Hippocampal replay in the awake state: A potential substrate for memory consolidation and retrieval. Nature Neuroscience, 14, 147-153.

Cheng, K., Shettleworth, S. J., Huttenlocher, J., \& Rieser, J. J. (2007). Bayesian integration of spatial information. Psychological Bulletin, 133, 625-637. http://dx.doi.org/10.1037/00332909.133.4.625.

Cheung, A., Ball, D., Milford, M., Wyeth, G., \& Wiles, J. (2012). Maintaining a cognitive map in darkness: The need to fuse boundary knowledge with path integration. PLoS Computational Biology, 8, e1002651.

Csizmadia, G., \& Muller, R. U. (2008). Storage of the distance between place cell firing fields in the strength of plastic synapses with a novel learning rule. Hippocampal Place Fields: Relevance to Learning and Memory: Relevance to Learning and Memory, 343.

Davachi, L., Mitchell, J. P., \& Wagner, A. D. (2003). Multiple routes to memory: Distinct medial temporal lobe processes build item and source memories. Proceedings of the National Academy of Sciences, 100, 2157-2162.

Derdikman, D., \& Moser, E. I. (2010). A manifold of spatial maps in the brain. Trends in Cognitive Sciences, 14, 561-569.

Diba, K., \& Buzsáki, G. (2007). Forward and reverse hippocampal place-cell sequences during ripples. Nature Neuroscience, 10, 1241-1242.

Dong, D., \& Franklin, S. (2015a). Modeling sensorimotor learning in LIDA using a dynamic learning rate. Biologically Inspired Cognitive Architectures, 14, 1-9. http://dx.doi.org/10.1016/j. bica.2015.09.005.

Dong, D., \& Franklin, S. (2015b). A new action execution module for the learning intelligent distribution agent (LIDA): The sensory motor system. Cognitive Computation, 1-17.

Durgin, F. H., Akagi, M., Gallistel, C. R., \& Haiken, W. (2009). The precision of locomotor odometry in humans. Experimental Brain Research, 193, 429-436.

Durrant-Whyte, H., \& Bailey, T. (2006). Simultaneous localization and mapping: Part i. Robotics \& Automation Magazine, IEEE, 13, 99-110.

Epstein, R. A. (2008). Parahippocampal and retrosplenial contributions to human spatial navigation. Trends in Cognitive Sciences, 12, 388-396.

Etienne, A. S., Maurer, R., \& Sguinot, V. (1996). Path integration in mammals and its interaction with visual landmarks. Journal of Experimental Biology, 199, 201-209.

Fiser, J., Berkes, P., Orbán, G., \& Lengyel, M. (2010). Statistically optimal perception and learning: From behavior to neura representations. Trends in Cognitive Sciences, 14, 119-130.

Fortin, N. (2008). Navigation and episodic-like memory in mammals (Vol. 1. Elsevier.

Franklin, S., Madl, T., D'Mello, S., \& Snaider, J. (2014). LIDA: A systems-level architecture for cognition, emotion, and learning. IEEE Transactions on Autonomous Mental Development, 6 , 19-41. http://dx.doi.org/10.1109/TAMD.2013.2277589.

Franklin, S., Strain, S., Snaider, J., McCall, R., \& Faghihi, U. (2012). Global workspace theory, its LIDA model and the underlying neuroscience. Biologically Inspired Cognitive Architectures.

Freeman, W. J. (2002). The limbic action-perception cycle controlling goal-directed animal behavior. Neural Networks, 3, 2249-2254.
Fuster, J. M. (2002). Physiology of executive functions: The perception-action cycle. Principles of Frontal Lobe Function, 96-108.

Glover, A. J., Maddern, W. P., Milford, M. J., \& Wyeth, G. F. (2010). Fab-map+ ratslam: Appearance-based slam for multiple times of day. In 2010 IEEE international conference on robotics and automation (pp. 3507-3512). IEEE.

Goertzel, B., Lian, R., Arel, I., de Garis, H., \& Chen, S. (2010). A world survey of artificial brain projects, Part ii: Biologically inspired cognitive architectures. Neurocomputing, 74, 30-49.

Gower, J. C. (1975). Generalized procrustes analysis. Psychometrika, 40, 33-51.

Grechkin, T. Y., Nguyen, T. D., Plumert, J. M., Cremer, J. F., \& Kearney, J. K. (2010). How does presentation method and measurement protocol affect distance estimation in real and virtual environments? ACM Transactions on Applied Perception, 7, 26.

Griffiths, T. L., Canini, K. R., Sanborn, A. N., \& Navarro, D. J. (2007). Unifying rational models of categorization via the hierarchical Dirichlet process. In Proceedings of the 29th annual conference of the cognitive science society (pp. 323-328).

Hafting, T., Fyhn, M., Molden, S., Moser, M., \& Moser, E. (2005). Microstructure of a spatial map in the entorhinal cortex. Nature, 436, 801-806.

Hartley, T., Maguire, E. A., Spiers, H. J., \& Burgess, N. (2003). The well-worn route and the path less traveled: Distinct neural bases of route following and wayfinding in humans. Neuron, 37, 877-888.

Hirtle, S., \& Jonides, J. (1985). Evidence of hierarchies in cognitive maps. Memory \& Cognition, 13, 208-217.

Jarsky, T., Roxin, A., Kath, W. L., \& Spruston, N. (2005). Conditional dendritic spike propagation following distal synaptic activation of hippocampal CA1 pyramidal neurons. Nature Neuroscience, 8, 1667-1676, <http://www.ncbi.nlm.nih.gov/ pubmed/16299501>.

Jeffery, K. J. (2007). Self-localization and the entorhinal-hippocampal system. Current Opinion in Neurobiology, 17, 684-691. http://dx.doi.org/10.1016/j.conb.2007.11.008.

Katz, Y., Kath, W. L., Spruston, N., \& Hasselmo, M. E. (2007). Coincidence detection of place and temporal context in a network model of spiking hippocampal neurons. PLoS Computational Biology, 3, e234.

Khaligh-Razavi, S., \& Kriegeskorte, N. (2014). Deep supervised, but not unsupervised, models may explain it cortical representation. PLoS Computational Biology, 10, e1003915.

Kiani, R., Esteky, H., Mirpour, K., \& Tanaka, K. (2007). Object category structure in response patterns of neuronal population in monkey inferior temporal cortex. Journal of Neurophysiology, 97, 4296-4309.

Kim, J., Delcasso, S., \& Lee, I. (2011). Neural correlates of objectin-place learning in hippocampus and prefrontal cortex. The Journal of Neuroscience, 31, 16991-17006.

Kjelstrup, K. B., Solstad, T., Brun, V. H., Hafting, T., Leutgeb, S., Witter, M. P., ... Moser, M. B. (2008). Finite scale of spatial representation in the hippocampus. Science, 321, 140-143.

Knill, D. C., \& Pouget, A. (2004). The Bayesian brain: The role of uncertainty in neural coding and computation. Trends in Neurosciences, 27, 712-719. http://dx.doi.org/10.1016/j. tins.2004.10.007.

Kravitz, D. J., Saleem, K. S., Baker, C. I., \& Mishkin, M. (2011). A new neural framework for visuospatial processing. Nature Reviews Neuroscience, 12, 217-230.

LeCun, Y., Bengio, Y., \& Hinton, G. (2015). Deep learning. Nature, $521,436-444$

Lever, C., Burton, S., Jeewajee, A., O Keefe, J., \& Burgess, N. (2009). Boundary vector cells in the subiculum of the hippocampal formation. Journal of Neuroscience, 29, 9771-9777. 
Madl, T., Chen, K., Montaldi, D., \& Trappl, R. (2015). Computational cognitive models of spatial memory in navigation space: A review. Neural Networks, 65, 18-43.

Madl, T., Franklin, S., Chen, K., Montaldi, D., \& Trappl, R. (2014). Bayesian integration of information in hippocampal place cells. PLoS ONE, e89762. http://dx.doi.org/10.1371/journal. pone.0089762.

Madl, T., Franklin, S., Chen, K., \& Trappl, R. (2013). Spatial working memory in the LIDA cognitive architecture. In Proceedings of the international conference on cognitive modelling.

Madl, T., Franklin, S., Chen, K., Trappl, R., \& Montaldi, D. (2016). Exploring the structure of spatial representations. PLOS ONE (submitted for publication). <http://madlnet.net/tamas/mapstructure.pdf >.

Manns, J. R., \& Eichenbaum, H. (2009). A cognitive map for object memory in the hippocampus. Learning \& Memory, 16, 616-624.

McCall, R., \& Franklin, S. (2013). Cortical learning algorithms with predictive coding for a systems-level cognitive architecture. In Second annual conference on advances in cognitive systems poster collection (pp. 149-66).

McNamara, T. P., Hardy, J. K., \& Hirtle, S. C. (1989). Subjective hierarchies in spatial memory. Journal of Experimental Psychology: Learning, Memory, and Cognition, 15, 211.

McNaughton, B., Barnes, C., Gerrard, J., Gothard, K., Jung, M., Knierim, J., ... Weaver, K. L. (1996). Deciphering the hippocampal polyglot: The hippocampus as a path integration system. The Journal of Experimental Biology, 199, 173-185.

McNaughton, B. L., Battaglia, F. P., Jensen, O., Moser, E. I., \& Moser, M. B. (2006). Path integration and the neural basis of the 'cognitive map'. Nature Reviews. Neuroscience, 7, 663-678. http://dx.doi.org/10.1038/nrn1932.

Mehta, M. R., Quirk, M. C., \& Wilson, M. A. (2000). Experiencedependent asymmetric shape of hippocampal receptive fields. Neuron, 25, 707-715.

Milford, M. J., Wiles, J., \& Wyeth, G. F. (2010). Solving navigational uncertainty using grid cells on robots. PLoS Computational Biology, 6, e1000995-1.

Milford, M. J., Wyeth, G. F., \& Rasser, D. (2004). Ratslam: A hippocampal model for simultaneous localization and mapping. In 2004 IEEE international conference on robotics and automation (pp. 403-408). IEEE.

Mittelstaedt, M., \& Mittelstaedt, H. (1980). Homing by path integration in a mammal. Naturwissenschaften, 67, 566-567.

Montemerlo, M., \& Thrun, S. (2007). FastSLAM: A scalable method for the simultaneous localization and mapping problem in robotics (Vol. 27). Springer.

Moser, E. I., Kropff, E., \& Moser, M. B. (2008). Place cells, grid cells, and the brain's spatial representation system. Annual Review of Neuroscience, 31, 69-89. http://dx.doi.org/10.1146/ annurev.neuro.31.061307.090723.

Murgia, A., \& Sharkey, P. M. (2009). Estimation of distances in virtual environments using size constancy. The International Journal of Virtual Reality, 8, 67-74.

Nardini, M., Jones, P., Bedford, R., \& Braddick, O. (2008). Development of cue integration in human navigation. Current Biology, 18, 689-693.

O'Keefe, J., \& Burgess, N. (1996). Geometric determinants of the place fields of hippocampal neurons. Nature, 381, 425-428.

Olson, E., Leonard, J., \& Teller, S. (2006). Fast iterative alignment of pose graphs with poor initial estimates. In Proceedings 2006 IEEE international conference on robotics and automation (pp. 2262-2269). IEEE.

Plumert, J. M., Kearney, J. K., Cremer, J. F., \& Recker, K. (2005). Distance perception in real and virtual environments. ACM Transactions on Applied Perception, 2, 216-233.

Prasser, D., Milford, M., \& Wyeth, G. (2006). Outdoor simultaneous localisation and mapping using ratslam. In Field and service robotics (pp. 143-154). Springer.
Quigley, M., Conley, K., Gerkey, B., Faust, J., Foote, T., Leibs, J., ...Ng, A. Y. (2009). Ros: An open-source robot operating system. In ICRA workshop on open source software (p. 5).

Rusu, R. B., Maldonado, A., Beetz, M., \& Gerkey, B. (2007). Extending player/stage/gazebo towards cognitive robots acting in ubiquitous sensor-equipped environments. In ICRA workshop for networked robot systems.

Samsonovich, A. V. (2010). Toward a unified catalog of implemented cognitive architectures. Biologically Inspired Cognitive Architectures, 221, 195-244.

Samsonovich, A. V. (2012). On a roadmap for the BICA challenge. Biologically Inspired Cognitive Architectures, 1, 100-107.

Samsonovich, A., \& McNaughton, B. L. (1997). Path integration and cognitive mapping in a continuous attractor neural network model. The Journal of Neuroscience, 17, 5900-5920.

Sanborn, A. N., Griffiths, T. L., \& Navarro, D. J. (2006). A more rational model of categorization. In Proceedings of the 28th annual conference of the cognitive science society (pp. 726731).

Schölkopf, B., \& Mallot, H. A. (1995). View-based cognitive mapping and path planning. Adaptive Behavior, 3, 311-348.

Snaider, J., \& Franklin, S. (2014). Modular composite representation. Cognitive Computation, 6, 510-527.

Snaider, J., McCall, R., \& Franklin, S. (2011). The LIDA framework as a general tool for AGI. In Artificial general intelligence (pp. 133-142). Springer.

Solstad, T., Boccara, C. N., Kropff, E., Moser, M. B., \& Moser, E. I. (2008). Representation of geometric borders in the entorhinal cortex. Science, 322, 1865-1868. http://dx.doi.org/ $10.1126 /$ science. 1166466.

Stork, D. G. (1989). Is backpropagation biologically plausible? In JCNN, international joint conference on neural networks (pp. 241-246). IEEE.

Strösslin, T., Sheynikhovich, D., Chavarriaga, R., \& Gerstner, W. (2005). Robust self-localisation and navigation based on hippocampal place cells. Neural Networks, 18, 1125-1140.

Szegedy, C., Liu, W., Jia, Y., Sermanet, P., Reed, S., Anguelov, D., .. Rabinovich, A. (2014). Going deeper with convolutions. Available from 1409.4842 .

Takahashi, H., \& Magee, J. C. (2009). Pathway interactions and synaptic plasticity in the dendritic tuft regions of CA1 pyramidal neurons. Neuron, 62, 102-111.

Taube, J. S. (2007). The head direction signal: Origins and sensorymotor integration. Annual Review of Neuroscience, 30, $181-207$.

Thrun, S., Burgard, W., \& Fox, D. (2005). Probabilistic robotics (intelligent robotics and autonomous agents). The MIT Press.

Thrun, S., \& Leonard, J. J. (2008). Simultaneous localization and mapping. Springer Handbook of Robotics, 871-889.

Vogeley, K., May, M., Ritzl, A., Falkai, P., Zilles, K., \& Fink, G. R. (2004). Neural correlates of first-person perspective as one constituent of human self-consciousness. Journal of Cognitive Neuroscience, 16, 817-827.

Waller, D. (1999). Factors affecting the perception of interobject distances in virtual environments. Presence: Teleoperators and Virtual Environments, 8, 657-670.

Williams, B., Cummins, M., Neira, J., Newman, P., Reid, I., \& Tardós, J. (2009). A comparison of loop closing techniques in monocular slam. Robotics and Autonomous Systems, 57, 1188-1197.

Wilson, D. I., Langston, R. F., Schlesiger, M. I., Wagner, M., Watanabe, S., \& Ainge, J. A. (2013). Lateral entorhinal cortex is critical for novel object-context recognition. Hippocampus, 23, 352-366.

Winters, B. D., \& Bussey, T. J. (2005). Transient inactivation of perirhinal cortex disrupts encoding, retrieval, and consolidation of object recognition memory. The Journal of Neuroscience, 25 , $52-61$. 
Yamins, D. L., Hong, H., Cadieu, C., \& DiCarlo, J. J. (2013). Hierarchical modular optimization of convolutional networks achieves representations similar to macaque it and human ventral stream. Advances in Neural Information Processing Systems, 3093-3101.
Zaehle, T., Jordan, K., Wüstenberg, T., Baudewig, J., Dechent, P., \& Mast, F. W. (2007). The neural basis of the egocentric and allocentric spatial frame of reference. Brain Research, 1137, 92-103. 